\title{
Percepção da população sul-mineira sobre o consumo e benefícios nutricionais da
}

\section{carne de coelho}

\author{
Perception of the sul-mineira population about the rabbit meat consumption and nutritional \\ benefits
}

Percepción de la población del sur de Minas Gerais sobre el consumo y los beneficios nutricionales

de la carne de conejo

Recebido: 15/06/2021 | Revisado: 23/06/2021 | Aceito: 28/06/2021 | Publicado: 12/07/2021

\author{
Mariely Simone Lopes Corrêa \\ ORCID: https://orcid.org/0000-0002-7032-1606 \\ Universidade José do Rosário Vellano, Brasil \\ E-mail: mariely_correa@hotmail.com \\ Andre Gomes Faria \\ ORCID: https://orcid.org/0000-0003-3364-1288 \\ Universidade José do Rosário Vellano, Brasil \\ E-mail: andre.faria@aluno.unifenas.br \\ Édina de Fátima Aguiar \\ ORCID: https://orcid.org/0000-0002-7883-5628 \\ Universidade José do Rosário Vellano, Brasil \\ E-mail: edina.aguiar@unifenas.br \\ Andressa Santanna Natel \\ ORCID: https://orcid.org/0000-0002-8252-1090 \\ Universidade José do Rosário Vellano, Brasil \\ E-mail: andressa.natel@unifenas.br \\ Valéria Quintana Cavicchioli \\ ORCID: https://orcid.org/0000-0001-5565-507X \\ Universidade José do Rosário Vellano, Brasil \\ E-mail: valeria.cavicchioli@unifenas.br \\ Danielly Beraldo dos Santos Silva \\ ORCID: https://orcid.org/0000-0002-3144-7476 \\ Universidade José do Rosário Vellano, Brasil \\ E-mail: danielly.silva@unifenas.br
}

\begin{abstract}
Resumo
A carne de coelho tem baixo teor de gordura, maiores teores de proteínas do que a carne bovina e suína. Além disso, a carne de coelho é rica em ômega 3, vitaminas do complexo B e minerais como potássio, ferro, fósforo e cálcio. No entanto, essa carne não é muito conhecida e consumida no Brasil. Portanto, este estudo teve por objetivo analisar a percepção da população sul mineira (Minas Gerais, Brasil) sobre a carne de coelho e os principais motivos que levam a aprovação ou rejeição da mesma. Para a realização desse estudo foi aplicado um questionário virtual. Como resultado, obteve-se 309 respostas de residentes de 44 cidades sul-mineiras. A partir da análise dos dados da pesquisa, foi possível definir quatro perfis de consumidores: I) nunca consumiram; II) tem vontade consumir; III) já consumiram uma ou duas vezes no ano; e IV) já consumiram mais de duas vezes por ano. A maioria dos entrevistados se enquadraram no perfil I e a minoria no perfil IV, o qual também foi caracterizado por consumidores que conhecem os benefícios nutricionais desta carne. Os resultados indicaram resistência por grande parte das pessoas em relação ao consumo de carne de coelho, isso se deve, na maioria das vezes por motivos culturais. Esses resultados poderá contribuir para o desenvolvimento de estratégias produtivas, colaborando para o aumento do consumo deste alimento.

Palavras-chave: Alimentação; Cunicultura; Mercado; Produção animal.
\end{abstract}

\begin{abstract}
Rabbit meat has a low-fat content, higher protein content than beef and pork. In addition, rabbit meat has high rates of omega 3, B vitamins and minerals such as potassium, iron, phosphorus and calcium. However, this meat is not widely known and consumed in Brazil. Therefore, this study aimed to analyze the consumer profile perception about rabbit meat in southern Minas Gerais (Brazil) and the main reasons for approval or disapproval. A virtual form was applied and 309 answers were obtained from residents of 44 cities in Minas Gerais. From the analysis was possible to define four consumer profiles: I) never consumed; II) wants to consume; III) have already consumed once or twice a year; and IV) have already consumed more than twice a year. Majority of the interviewees were profile I and minority were profile IV, which is also characterized by consumers who know about rabbit meat nutritional benefits. The results
\end{abstract}


indicated resistance by most people in relation to the rabbit meat consumption, probably this is due the cultural reasons. These results contribute to the development of productive strategies, helping to increase the consumption of this food.

Keyword: Food; Rabbit farming; Market; Animal production.

\section{Resumen}

La carne de conejo es baja en grasa, más alta en proteínas que la carne de res y cerdo. Además, la carne de conejo es rica en omega 3, vitaminas del grupo B y minerales como potasio, hierro, fósforo y calcio. Sin embargo, esta carne no es muy conocida y consumida en Brasil. Por tanto, este estudio tuvo como objetivo analizar la percepción del perfil de los consumidores sobre la carne de conejo en el sur de Minas Gerais (Brasil) y las principales razones que conducen a su aprobación o rechazo. Para la realización de este estudio se aplicó un cuestionario virtual. Se obtuvieron 309 respuestas de residentes de 44 ciudades del sur de Minas Gerais. A partir del análisis de los datos de la encuesta, fue posible definir cuatro perfiles de consumidores: I) nunca consumí; II) quiere consumir; III) ya lo han consumido una o dos veces al año; y IV) ya han consumido más de dos veces al año. La mayoría de los entrevistados encajan en el perfil I y una minoría en el perfil IV, que también se caracteriza por consumidores que conocen los beneficios nutricionales de esta carne. Los resultados indicaron resistencia de la mayoría de las personas en relación al consumo de carne de conejo, esto se debe, en la mayoría de los casos, a razones culturales. Estos resultados contribuyen al desarrollo de estrategias de producción, contribuyendo al aumento del consumo de este alimento.

Palabras clave: Alimento; Producción de conejos; Mercado; Producción animal.

\section{Introdução}

A carne de coelho possui muitos dos nutrientes considerados fundamentais para a qualidade de vida dos seres humanos (Dalle Zotte, Szendrő, 2011; Jia et al., 2017; Lauková et al., 2017). Nesse sentido, Dalle Zotte e Szendrő (2011) mostraram que a carne de coelho é considerada "alimento funcional", caracterizado pela contribuição positiva para uma ou mais funções do organismo humano. O consumo da carne de coelho é condizente com novos padrões de hábitos alimentares que estimulam e valorizam os alimentos com baixos teores sódio e colesterol. A mudança no estilo de vida da sociedade ocorreu devido as condições econômicas que propiciaram a evolução alimentar com produtos de maior qualidade (Priyanti e Raharjo, 2012). A carne de coelho também proporciona retorno financeiro, já que é um produto de maior valor agregado e que atende a mercados mais exigentes.

Segundo Malavé et al., (2013), a cunicultura é uma importante alternativa de produção adaptável aos ambientes adversos, pois consegue ser desenvolvida em vários países do mundo, uma vez que, a produção pode ser reajustada à realidade socioeconômica de qualquer região ou comunidade (Nieves et al., 2009). A criação de coelhos pode ser uma alternativa para diversificar a produção de pequenos produtores e obter maior renda. Além disso, o consumo de carnes está atrelado a redução dos impactos ambientais decorrentes da produção dos animais. Neste quesito, a cunicultura se destaca, uma vez que, é considerada um dos setores mais sustentáveis e simples, promovendo uma tendência de crescimento da produção (Valverde et al., 2021).

A região sul do Brasil tem a maior produção de coelhos do País, visando a exportação. Estados como São Paulo e Rio de Janeiro, nestes últimos anos, apresentaram maior demanda, assim como o mercado do Nordeste (Valeverde et al., 2021). Em 2012, segundo o IBGE (últimos dados disponíveis), a produção de coelhos no Estado de Minas Gerais, tinha um volume anual de 14,8 mil cabeças, correspondendo 7,2\% do volume nacional. Esses dados mostraram a grande expansão da cunicultura no Estado, porém, o seu consumo ainda é bastante limitado. Um dos motivos são os fatores psicográficos, os quais estão envolvidos nos comportamentos individuais a partir do ambiente a que estão inseridas, ou seja, estilo de vida, valores culturais e valores pessoais (Buitrago -Vera et al., 2016).

O consumo de carne de coelho ainda não é comum e é pouco inserida na cultura alimentar dos mineiros. No entanto, existe a tendência de maior demanda nos próximos anos. Para que os inúmeros entraves existentes dentro da cadeia produtiva da cunicultura sejam superados, são necessárias estudos e práticas de marketing que buscam conhecer as necessidades do mercado, características e comportamentos (Buitrago-Vera et al., 2016). Diante disso, existe a necessidade de identificação do 
interesse de consumo da carne de coelho para entender os motivos que levam a aprovação ou rejeição. Portanto, o objetivo deste estudo foi analisar a percepção dos consumidores sobre a carne de coelho como alimento e verificar se há possibilidade de expansão da comercialização desse produto nas cidades do sul de Minas Gerais.

\section{Metodologia}

Este estudo caracteriza-se por uma pesquisa de opinião pública com participantes não identificados, portanto segundo a Resolução no 510/2016, é dispensado a avaliação do CEP/CONEP (Brasil, 2016). A pesquisa foi conduzida por meio de um estudo de corte transversal, de caráter descritivo, individualizado, observacional e estatístico. A coleta de dados ocorreu por meio de um questionário virtual - Google Forms (Formulários do Google) que buscava compreender a percepção dos consumidores sobre a carne de coelho como alimento (Tabela 1). O questionário ficou disponível para acesso da população de agosto à dezembro de 2018. Para a divulgação e abordagem do instrumento, foram divulgados links do questionário em diversos ambientes virtuais como: mídias acadêmicas e sociais.

Após o término da coleta das respostas, os dados foram exportados para uma planilha e foram excluídas todas as respostas provenientes de participantes moradores em outros Estados e municípios que não pertenciam à região do Sul de Minas Gerais. Os dados foram analisados com o auxílio dos recursos de análise de dados do Excel. A análise dos dados permitiu a identificação de padrões que melhor definiram o perfil do consumidor (ativo e potencial) de carne de coelho no Sul de Minas Gerais. Os dados permitiram ainda uma investigação de possíveis perspectivas do setor para cada perfil com o intuito de fomentar maiores informações sobre o comportamento dos agentes envolvidos na pesquisa.

Tabela 1 - Questionário virtual. Perguntas realizadas para melhor compreensão da percepção dos consumidores sobre a carne de coelho como alimento na região Sul de Minas Gerais, Brasil.

\section{Informações Gerais}

1. Idade.

2. Cidade/Estado em que vive atualmente.

3. Escolaridade.

5. Sexo.

6. Remuneração familiar.

7. Onde realiza suas refeições?

8. Por que faz suas refeições nesse lugar?

\begin{tabular}{l}
\hline Informações de Consumo \\
\hline 9. Já comeu carne de coelho ao menos uma vez? \\
10. Se não, por que nunca comeu carne de coelho? \\
11. Se não, gostaria de experimentar carne de coelho? \\
12. Se sim, no período de 1 ano, quantas vezes você normalmente consome carne de coelho? \\
13. Se não, gostaria de experimentar carne de coelho? \\
14. Conhece os benefícios nutricionais da carne de coelho para a saúde humana? \\
\hline
\end{tabular}

Fonte: Autores.

\section{Resultados e Discussão}

\subsection{Caracterização dos participantes da pesquisa}

No total, foram coletadas 383 respostas de residentes em 8 estados e 86 cidades. As respostas provenientes de Estados e municípios que não pertenciam à região do Sul de Minas Gerais foram excluídas. Sendo assim, foram excluídas 73 respostas, permanecendo 309 respostas. Considerando a população total sul-mineira (N=2.944.681 - IBGE, 2020) e as 309 respostas 
obtidas, a pesquisa teve margem de erro de $5.57 \%$ à $95 \%$ de confiabilidade. Do total de 155 cidades do Sul de Minas Gerais (IBGE, 2020), 44 cidades (28\%) estavam incluídas nesta pesquisa (Tabela 2).

Tabela 2 - Cidades sul-mineiras incluídas na pesquisa de opinião para compreender a percepção dos consumidores sobre a carne de coelho como alimento.

\begin{tabular}{llll}
\hline \multicolumn{3}{c}{ Cidades sul-mineiras } \\
\hline Aguanil & Campos Gerais & Itajubá & Passos \\
Alfenas & Careaçu & Itumirim & Pedralva \\
Alterosa & Carmo da Cachoeira & Juruaia & Piranguinho \\
Areado & Carmo do Rio Claro & Lambari & Poço Fundo \\
Bandeira do sul & Divisa Nova & Lavras & Poços de caldas \\
Borda da mata & Elói Mendes & Machado & Pouso Alegre \\
Cabo Verde & Extrema & Muzambinho & Santa Rita do Sapucaí \\
Caldas & Guapé & Natércia & São Gonçalo do Sapucaí \\
Cambuí & Guaranésia & Nepomuceno & São João da Mata \\
Campanha & Guaxupé & Nova Resende & Três Pontas \\
Campestre & Ijaci & Paraguaçu & Varginha \\
\hline
\end{tabular}

Fonte: Autores.

As faixas etárias apresentadas em anos dos participantes foram predominantes as idades entre 16 e 30 anos (N=209), seguida da faixa etária entre 31 e 45 anos (N=74). Também participaram da pesquisa, 24 pessoas com idade entre 46 e 60 anos; 2 pessoas com idade inferior a 15 anos e nenhum com idade de 61 anos ou mais. Esses resultatos indicam, principalmente que a faixa etária de de 61 anos ou mais (idosos), tem uma baixa inclusão tecnológica (Luo e Menec, 2018).

Aproximadamente $67 \%$ dos participantes são do sexo feminino e $33 \%$ do sexo masculino. A remuneração familiar mais citada pelos entrevistados foi a que contemplou valores entre 2 e 3 salários mínimos (27\% dos participantes), seguidas pelas famílias que possuem mais de 5 salários mínimos (24\%). Rendas entre 1 e 2 salários mínimos representaram 23\% da população e $19 \%$ possuíam rendas entre 3 e 4 salários mínimos. Apenas $6 \%$ dos entrevistados responderam ter renda familiar inferior a 1 salário mínimo. O nível de escolaridade dos entrevistados o que predominou foi de pessoas que possuíam ensino médio completo ou curso superior concluído. Juntas, essas duas categorias representaram 78\% do campo amostral.

Em relação a alimentação, aproximadamente $77 \%$ das pessoas responderam que realizam suas refeições em seus domicílios. Os mesmos justificaram que, predominantemente, as refeições são realizadas em seus lares por gostarem; por não terem outra alternativa ou por falta de tempo. O percentual de pessoas que se alimentam em restaurantes e no trabalho foi de $12 \%$ e $11 \%$, respectivamente. A justificativas predominantes foram falta de tempo e por falta de opção.

As cidades sul-mineiras, na maioria, são consideradas pequenas e de interior. Esse fato, colabora para a fácil e rápida locomoção das pessoas, facilitando a realização das refeições principais em seus domicílios, diferente de grandes centros urbanos. Além disso, existe a cultura das pessoas se reunirem com seus familiares para realizarem suas refeições principais do dia (Fortunato e Vicenzi, 2018).

\subsection{Perfil do consumo da carne de coelho}

A partir da análise dos dados da pesquisa, foi possível definir quatro perfis de consumidores. Os perfis foram apresentados na Tabela 3 . 
Tabela 3 - Perfil dos entrevistados na pesquisa de opinião para comprender a percepção dos consumidores sobre a carne de coelho como alimento no Sul de Minas Gerais, Brasil.

\begin{tabular}{|c|c|c|c|c|}
\hline & Perfil I & Perfil II & Perfil III & Perfil IV \\
\hline $\begin{array}{l}\text { Número de } \\
\text { Pessoas }\end{array}$ & 119 pessoas $(39 \%)$ & 70 pessoas $(22 \%)$ & 111 pessoas $(36 \%)$ & 9 pessoas $(3 \%)$ \\
\hline Consumo & $\begin{array}{c}\text { Nunca consumiu } \\
\text { carne de coelho e não } \\
\text { gostaria }\end{array}$ & $\begin{array}{c}\text { Nunca consumiu } \\
\text { carne de coelho, mas } \\
\text { gostaria }\end{array}$ & $\begin{array}{l}\text { Consome carne de } \\
\text { coelho com pouca } \\
\text { frequência }\end{array}$ & $\begin{array}{l}\text { Consome carne de } \\
\text { coelho com mais } \\
\text { frequência. }\end{array}$ \\
\hline Perfil & $\begin{array}{l}\text { Desconhecem os } \\
\text { benefícios de } \\
\text { consumo e não } \\
\text { associam a carne de } \\
\text { coelho para a } \\
\text { alimentação. }\end{array}$ & $\begin{array}{l}\text { Desconhecem os } \\
\text { benefícios de } \\
\text { consumo e } \\
\text { desconhecem pontos } \\
\text { de venda da carne de } \\
\text { coelho. }\end{array}$ & $\begin{array}{l}\text { Possuem certo grau } \\
\text { de conhecimento } \\
\text { sobre os benefícios de } \\
\text { consumo, mas comem } \\
\text { carne de coelho } \\
\text { poucas vezes por ano. }\end{array}$ & $\begin{array}{c}\text { Maior } \\
\text { conhecimento sobre } \\
\text { os benefícios do } \\
\text { consumo com } \\
\text { possibilidade de } \\
\text { expansão de } \\
\text { consumo. }\end{array}$ \\
\hline Perspectiva & $\begin{array}{c}\text { Não consumirá carne } \\
\text { de coelho }\end{array}$ & $\begin{array}{c}\text { Se houver } \\
\text { oportunidade, } \\
\text { consumirá carne de } \\
\text { coelho }\end{array}$ & $\begin{array}{l}\text { Aumentará a } \\
\text { frequência de } \\
\text { consumo, se } \\
\text { incentivados. }\end{array}$ & $\begin{array}{l}\text { Permanecerá } \\
\text { consumindo }\end{array}$ \\
\hline
\end{tabular}

Fonte: Autores.

A maior parte dos participantes nunca experimentaram carne de coelho (61\%). Destes, a maioria (39\%) responderam não ter interesse algum em experimentar a carne proveniente da cunicultura de corte, o que caracteriza o perfil I. Esses resultados apontam que a população sul mineira tem preferência pelo tradicionalismo alimentar. Contudo, os resultados sugerem que não se trata de uma decisão apoiada no conhecimento, mas em hábitos, crenças e valores sobre a carne de coelho (Silva et al., 2020).

O perfil I também foi caracterizado por indivíduos que não conhecem informações sobre os benefícios que o consumo regular da referida carne pode trazer para a saúde humana. Além disso, alguns dos participantes não consideraram a carne de coelho como alimento, por terem sentimentos correlatos ao animal. Giumelli e Santos (2016) afirmaram que esse sentimento de compaixão para com os coelhos se dá devido ao aumento expressivo do mercado de coelhos como animais de estimação. Os principais fatores aliados ao consumo de alimentos são os fatores psicográficos que norteiam comportamentos individuais nas pessoas a partir do ambiente a que estão inseridas, ou seja, estilo de vida e os valores pessoais como elementos que contribuem para sua aceitação (ou não) (Buitrago-Vera et al., 2016). Os dados indicaram que dificilmente as pessoas com essas características consumirão carne de coelho à curto prazo, demostrando que para esse perfil, o mercado da cunicultura ainda não é atrativo.

O perfil II foi caracterizado pelo público que nunca consumiu a carne de coelho e que gostaria de consumi-la, aproximadamente $22 \%$ manifestaram esse desejo. Esses consumidores, responderam que não se alimentaram por falta de oportunidade de comprar esse produto em restaurantes, supermercados, outros estabelecimentos de comercialização de alimentos ou pelo preço elevado. Portanto, é possível depreender que um dos fatores que não estimulam o consumo da carne de coelho da região sul mineira é a carência desse produto para a comercialização nos principais estabelecimentos das cidades.

Os estados que mais produzem carne de coelho com foco na exportação, são os da região sul (com pouco mais de 119 mil cabeças). Na região sudeste, o Estado que mais produz é São Paulo, onde há maior demanda por carnes exóticas. Em Minas Gerais, a criação de coelhos é baixa em relação ao potencial do Estado e um dos principais problemas é a falta do hábito de consumo da carne de coelho, que não está inserido na cultura alimentar, como já discutido anteriormente (Valverde, 2021).

Os consumidores do perfil II desconheciam os benefícios que o consumo da carne de coelho pode oferecer à saúde 
humana. Tal indicador demonstra que, as pessoas que gostaria de experimentar essa carne, anseiam em consumi-la por curiosidade. Para esse perfil de consumidor, existe a necessidade de estudos e investimentos em ações que promovam e estimulem o consumo, e principalmente, a percepção do benefício nutricional desse produto no mercado sul-mineiro. Com políticas de marketing e disponibilidade de mercado, o consumo de carne de coelho poderá aumentar com o tempo e os valores diminuírem. Azevedo, Araújo e Duarte (2017) mostraram que as estratégias de marketing são mais eficientes se todas as informações forem utilizadas como vantagem competitiva.

O Perfil III foi formado por 111 pessoas que consumiram carne de coelho, porém com baixa frequência. Os resultados indicam maior expressividade sobre o conhecimento das pessoas em relação as contribuições que o consumo dessa carne pode oferecer à saúde. O perfil III possui características mais fáceis de serem utilizadas como impulsionadoras do aumento no consumo de carne de coelho. Uma das estratégias seriam a a maior disponibilidade dessa carne em restaurantes ou outros estabelecimentos alimentícios nas cidades sul-mineiras. Também notou-se que praticamente todos os entrevistados não possuem a percepção do coelho como animal de estimação. Esse resultado, possivelmente, está associado à baixa popularidade e valores altos da carne, mesmo entre pessoas que já experimentaram, ainda têm-se pouca demanda pela carne de coelho no sul de Minas Gerais por esse perfil de consumidores (Valverde, 2021).

O perfil IV foi caracterizado por pessoas ( $\mathrm{N}=9,3 \%$ dos entrevistados) que consumiram carne de coelho com maior frequência. Neste perfil de consumidores também foi notado maior rentabilidade familiar. No Brasil, grande parte da produção de carne de coelho é voltada para o mercado "Classe A", o que justifica o alto preço desta carne nos comércios (Valverde, 2021). Outra característica que define esse grupo é o alto percentual de pessoas que conhecem os benefícios em se consumir carne de coelho. Portanto, esse perfil demostra que não só consomem a carne por gosto, como também por benefícios à saúde. Os consumidores do perfil IV afirmaram que continurão consumindo a carne no futuro, o que demostra que cunicultura continuará com demanda para ofertar produtos advindo da carne, seja in natura ou processada para estes consumidores.

O consumo de carnes considerada exóticas, como a do coelho, no Brasil ainda é baixo. No entanto, existe uma tendência para expansão a qual promoverá, não somente o desenvolvimento econômico, como também a preservação das raças regionais (Amaral et al., 2016; Silva et al., 2020). Considerando a avaliação e identificação do perfil de consumidores para carne, a criação de coelhos pode ser uma boa opção para diversificar a produção e obter maior renda, principalmente, para pequenos produtores sul-mineiros.

Como os animais são de fácil manejo, a tendência é que produção seja aumentada. Além disso a alta produtividade e ciclo curto faz com que a cunicultura pode ser desenvolvida juntamente com outras atividades, sendo uma alternativa para o produtor que cria caprinos, suínos, bovinos ou outros animais (Silva et al., 2020). Mesmo que o consumo da carne de coelho não faça parte da cultura alimentar dos sul-mineiros, a produção regional e nacional de coelhos está em crescimento. A tendência é de que haja maior demanda e redução dos valores nos próximos anos, uma vez os animais tem fácil manejo e a carne tem benefícios nutricionais consideráveis.

\section{Conclusão}

Essa pesquisa mostrou o perfil de consumidores de carne de coelho no sul de Minas Gerais e os principais motivos que levam a aprovação ou rejeição da mesma. Esses resultados contribuem para a expansão e criação de estratégias de produção e consumo deste alimento. Embora os resultados tenham indicado muita resistência por grande parte das pessoas, também permitem inferir que o mercado de carne de coelho sul-mineiro está em amplo crescimento. 


\section{Agradecimentos}

À Universidade José do Rosário Vellano. O presente trabalho foi realizado com apoio da Coordenação de Aperfeiçoamento de Pessoal de Nível Superior - Brasil (CAPES) - Código de Financiamento 001 e do Conselho Nacional de Desenvolvimento Científico e Tecnológico - Brasil (CNPq).

\section{Referências}

Abdel-Wareth, A. A., Taha, E. M., Südekum, K. H., \& Lohakare, J. (2018). Thyme oil inclusion levels in a rabbit ration: Evaluation of productive performance, carcass criteria and meat quality under hot environmental conditions. Anim Nutr, 4, 410-416.

Acuña, A. M., Córdova, L., García, A., \& Méndez, J. (2013). Composición bromatológica de la carne de conejos suplementados con mataratón y cachaza de palma aceitera. Rev.MVZ Córdoba, 18(2), 3452-3458.

Amaral, A. G., Campos, J. C. D., Ramos, T. V., Taveira, R. Z., Silveira Neto, O. J., Gomes, R. C. C., Pereira, K. A., \& Oliveira, O. A. M. (2016). Avaliação de mercado de carnes de animais silvestres e exóticos na Região Metropolitana de Goiânia. Revista ESPACIOS, 37(14).

Azevedo, A. W., De Araújo, W. J., \& Duarte, E. N. (2017). Prospecção de cenários para competências em informação como instrumento de inteligência competitiva. InCID, 8 (2), 42-68.

Brasil. Resolução n ${ }^{\circ}$ 510, de 07 de abril de 2016. Dispõe sobre as normas aplicáveis a pesquisas em Ciências Humanas e Sociais. Diário Oficial [da] República Federativa do Brasil, Brasília, DF, 24 maio 2016. Disponível em: 〈Disponível em: http://bit.ly/2fmnKeD >. Acesso em: 20 jun. 2016.

Buitrago-Vera, J., Escriba-Perez, C., Baviera-Puig, A., \& Montero-Vicente, L. (2016). Consumer segmentation based on food-related lifestyles and analysis of rabbit meat consumption. WRSA, 24(3), 169-182.

Cavani C., Petracci M., Trocino A., \& Xiccato G. (2009). Advances in research on poultry and rabbit meat quality. Ital. J. Anim. Sci, 8(2), 741-750.

Dalle Zotte, A., \& Szendrő, Z. (2011). The role of rabbit meat as functional food. Meat Sci, 88(3), 319-331, 2011.

Fortunato, L. H., \& Vicenzi, K. (2018). Conhecimento sobre prática de higiene na manipulação de alimentos em residências de Caxias do Sul-RS. UNINGÁ $\operatorname{Rev}, 17(1)$.

Giumelli, R. D., \& Santos, M. C. P. (2016). Convivência com animais de estimação: um estudo fenomenológico. Rev. Abordagem Gestált, 22(1), 49-58.

Gómez-Salazar, J. A., Ochoa-Montes, D. A., Cerón-García, A., Ozuna, C., \& Sosa-Morales, M. E. (2018). Effect of acid marination assisted by power ultrasound on the quality of rabbit meat. J Food Qual., 2018.

González-Redondo, P., \& Contreras-Chacón, G. M. (2020). Perceptions among university students in Seville (Spain) of the rabbit as livestock and as a companion animal. World Rabbit Sci, 20(3), 155-162, 2012.

IBGE. (2020). https://www.ibge.gov.br/cidades-e-estados/mg.html

Jia, G., Liuc, H., Nirasawad, S., \& Liu, H. (2017). Effects of high-voltage electrostatic field treatment on the thawing rate and post-thawing quality of frozen rabbit meat. Innov Food Sci Emerg Technol, 41, 348-356, 2017.

Kotler, P., Armstrong, G. (2015). Principles of marketing. (16a ed.), Prentice Hall: Upper Saddle River NJ, USA.

Las Casas, A. L., Bacha, R. A. F., \& Carvalho, C. M. E. (2016). O agronegócio e o marketing rural no estado de Mato Grosso do Sul. REHR, 29 (55).

Lauková, A., Szabóová, R., Pleva, P., Buňková, L., \& Chrastinová, L. (2017). Decarboxylase-positive Enterococcus faecium strains isolated from rabbit meat and their sensitivity to enterocins. Food Sci Nutr, 5 (1), 31-37.

Lukefahr, S. D., Cheeke, P. R., Mcnitt, J. I., \& Patton, N. M. (2004). Limitations of intensive meat rabbit production in North America: A review. Can. J. Anim. Sci, 84(3), 349-360.

Luo, H., \& Menec, V. (2018). Social Capital and Health Among Older Chinese Immigrants: a Cross-Sectional Analysis of a Sample in a Canadian Prairie City. J Cross Cult Gerontol, 33(1), 65-81.

Nieves, D., Terán, O., Vivas, M., Arciniegas, G., \& González, C. (2009). Comportamiento productivo de conejos alimentados con dietas basadas en follajes tropicales. Rev Científ FCV-LUZ Rev. 21(2), 173-180.

Petracci, M., \& Cavani, C. (2013) Rabbit meat processing: historical perspective to future directions. World Rabbit Sci, 21 (4), $217-226$.

Priyanti, A., \& Raharjo, Y. C. (2012). Market driving to develop rabbit meat products In Indonesia. Wartazoa. 22(3), 99-106.

Sánchez-Laiño, A., Torres-Navarrete, E. D., Buste- Castro, F., Barrera-Álvarezand, A., \& Sánchez-Torres, J. (2018). Forrajes tropicales como alternativa alimenticia en conejos de engorde (Oryctolagus cuniculus L.). AcAg, 67 (2), 333-338.

Silva, B. P., Bassiga, B. A., Silva Ferreira, M. F. D. S., Carniatto, C. H. O., Feitosa, L. G. A., Batista, A. E. L., Tormem, F., \& Correa, V. G. (2020). Consumo de Carne de Coelho: Aspectos Culturais e Sensoriais. Brazilian Journal of Development, 6(11), 93361-93371.

Swatland, H. J. (2010). Meat products and consumption culture in the West. Meat Sci. 86(1), 80-85.

Valverde, M. (2021). Cunicultura é boa opção para produtor - Diário do Comércio https://diariodocomercio.com.br/agronegocio/cunicultura-e-boa-opcao-paraprodutor. 